\title{
Aumento Gingival en Base a Ácido Hialurónico en Defectos Perimplantares y Periodontales. Análisis de una Serie de Casos
}

\author{
Gingival Increase Based on Hyaluronic Acid in Peri Implant \\ and Periodontal Defects. A Case Series Analysis
}

\author{
Antônio Celória'; Eder Alberto Sigua-Rodriguez² \& Sergio Olate ${ }^{3}$
}

CELÓRIA, A.; SIGUA-RODRIGUEZ, E. A. \& OLATE, S. Aumento gingival en base a ácido hialurónico en defectos perimplantares y periodontales. Análisis de una serie de casos. Int. J. Odontostomat., 11(4):431-435, 2017.

RESUMEN: El ácido hialurónico ha sido empleado en diferentes áreas anatómicas, su aplicación para el rejuvenecimiento facial y en las lesiones de la articulación son conocidos; sin embargo, la aplicación en defectos periodontales y perimplantares tiene limitada información. El objetivo de esta investigación es mostrar los resultados clínicos de una serie de casos de sujetos con defectos periodontales menores tratados con la aplicación de ácido hialurónico. Se incluyeron 4 sujetos que presentaron espacios negros y recesiones gingivales en los que se aplicó el protocolo de instalación de ácido hialurónico al 0,2 \% en diferentes momentos; el seguimiento clínico demostró eficiencia en la recuperación estética y estabilidad en los aumentos. Es posible concluir que esta técnica mínimamente invasiva es promisoria para tratar defectos periodontales y perimplantares menores.

PALABRAS CLAVE: ácido hialurónico, estética implantaria, defecto periodontal.

\section{INTRODUCCIÓN}

La recesión gingival es una condición muy común, su extensión y prevalencia aumenta con la edad, se estima que alrededor del $50 \%$ de la población tiene uno o más sitios con $1 \mathrm{~mm}$ o más con exposición radicular y puede aumentar hasta un $88 \%$ en las personas mayores de 65 años (Kassab \& Cohen, 2003).

Estas recesiones pueden causar pérdidas de la papila interdentaria, determinando la presencia de espacios negros (black spaces), presentes en más de un tercio de los adultos. Además de generar deficiencias estéticas, retenciones de alimentos y algunas alteraciones fonéticas, se genera una total afectación de la salud de los tejidos periodontales (Celória, 2015). La rehabilitación basada en prótesis fija soportado por implantes dentarios también sufre estos problemas de recesiones con exposición de las cintas metálicas de los implantes.
En la exposición cervical del implante y el manejo de alteraciones perimplantarias algunas intervenciones quirúrgicas han sido eficientes, sin embargo, la morbilidad y el costo puede limitar su indicación. Otras alternativas no quirúrgicas han sido empleadas; Ballini et al. (2009) indicaron que el uso del ácido hialurónico promueve la regeneración y la estabilidad tisular para la cobertura radicular; además, se ha sugerido a partir de estudios en animales (Aslan et al., 2006) que podría existir un aumento en el componente de tejido duro del periodonto después del tratamiento, aunque los resultados no son concluyentes.

En este sentido, Ferguson et al. (2011) indicaron que el empleo de ácido hialurónico permite mayor rapidez en la cicatrización de las heridas debido a la mayor migración y proliferación celular, facilitando la infiltración de glóbulos blancos junto a la hidratación

\footnotetext{
${ }^{1}$ CEODAC Pós-graduação em odontologia - Maringá - Paraná - Brasil.

${ }^{2}$ Centro de Investigaciones del Colegio Odontológico (CICO), Institución Universitaria Colegios de Colombia - Bogotá - Colombia.

${ }^{3}$ División de Cirugía Oral, Facial y Maxilofacial. Centro de Excelencia en Estudios Morfológicos y Quirúrgicos, Universidad de La Frontera, Temuco, Chile.
} 
de estos tejidos, lo que podría contribuir a una mejor estabilidad de los procedimientos de cobertura radicular.

El objetivo de este artículo es presentar la experiencia inicial de los autores con una serie de 4 casos de corrección de espacios negros y recesiones gingivales con el uso de ácido hialurónico.

\section{PROTOCOLO DE TRATAMIENTO}

Todos los pacientes incluidos firmaron un documento de consentimiento informado para esta investigación. El protocolo clínico instaurado implica inicialmente salud periodontal en ausencia de procesos inflamatorios locales a fin de tener la base para la aplicación de los productos.

Posteriormente es realizado el procedimiento sobre anestesia local mediante bloqueo con técnica troncular o infiltrativa, distante de la región que será aplicado el ácido, para evitar alteraciones en su morfología y vasoconstricción del tejido. Posteriormente, se realiza la aplicación de ácido hialurónico $(\mathrm{AH})$ al 0,2 \%; cuando es aplicado en la papila, el lugar adecuado es a $3 \mathrm{~mm}$ del vértice de la papila y en el caso de ser realizado a nivel cervical del implante se debe realizar una leve movilización del surco gingival previamente; es posible en esta fase realizar la aplicación en dos a tres regiones diferentes, dependiendo del tamaño del defecto, realizando las punciones $2 \mathrm{~mm}$ debajo del surco gingival; la técnica se debe realizar con una infiltración lenta, analizando la isquemia de la papila, este será muestro mejor parámetro para finalizar la aplicación; la cantidad de $\mathrm{AH}$ aplicado se relaciona al tamaño del defecto y la cantidad de tejido que se requiere modificar.

El seguimiento de los pacientes en la presente serie fue realizado en 6 oportunidades posteriores a la primera aplicación, incorporando una nueva aplicación si se consideraba necesaria a los 21 días; como medicación postoperatoria sólo se utilizan analgésicos en caso de dolor.

Los autores del presente estudio recomiendan realizar la documentación fotográfica en cada consulta y también en las aplicaciones posteriores y así poder comparar los resultados alcanzados. Nuestra experiencia nos ha mostrado que una nueva aplicación podría estar indicada con 21 días después la primera aplicación.

\section{SERIE DE CASOS}

Caso 1: Paciente de sexo femenino, 21 años, ASA I, posterior a la finalización del tratamiento ortodóncico presentó un espacio negro en la zona entre dientes 11 y 12 con quejas funcionales y estéticas. Fue realizada nuestro protocolo con la aplicación de AH (TEOSYAL $®$ ) en dos oportunidades con un total de $0,2 \mathrm{~mL}$ en cada aplicación utilizando intervalos de 21 días. La paciente presentó resultados satisfactorios estéticos y funcionales, actualmente se encuentra con 4 años de seguimiento y no ha mostrado ninguna señal de recidiva (Fig. 1).

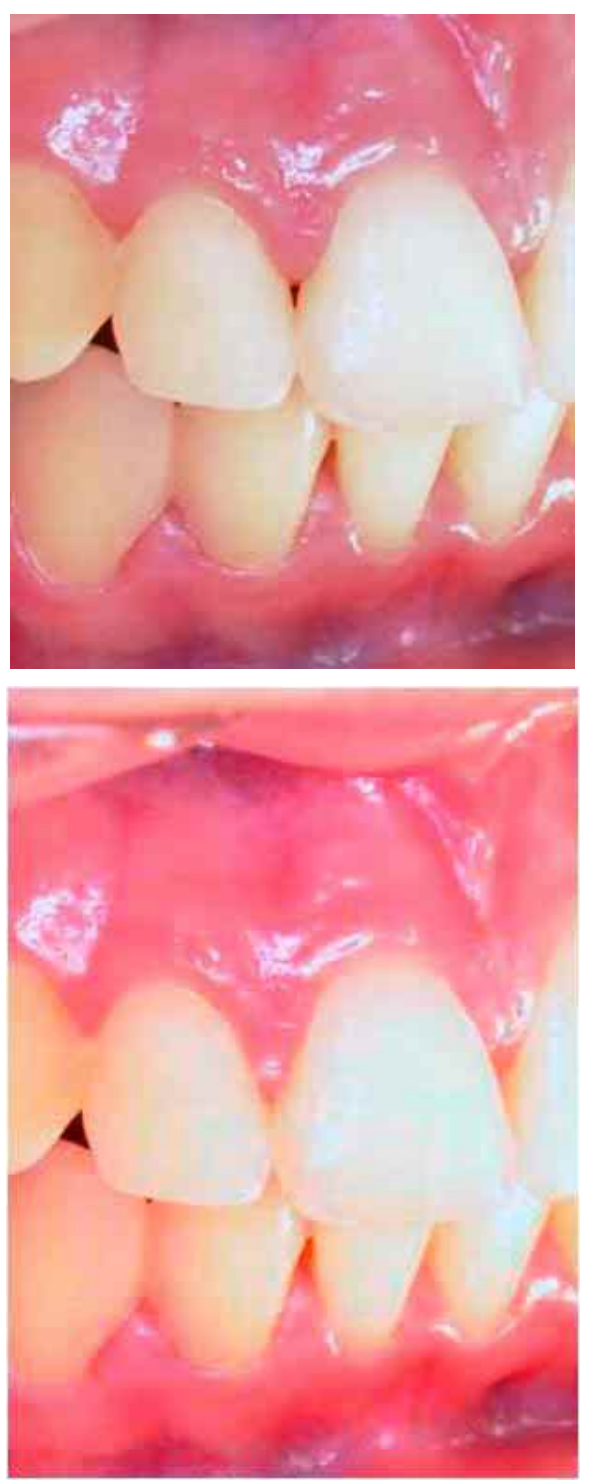

Fig. 1. A) Presencia de espacio negro entre incisivo central y lateral. B) Imagen con 1 año de seguimiento. 
Caso 2: Paciente de sexo masculino 59 años, ASA I, presentaba quejas estéticas por presentar un espacio negro en la región entre 11 y 12 . Fue realizada el protocolo aplicando $0,2 \mathrm{~mL}$ de AH (TEOSYAL $®$ ) en 2 oportunidades con intervalos de 21 días. Actualmente el paciente se encuentra con 2 años de seguimiento con resultados satisfactorios estéticos y funcionales, sin señal de recidiva (Fig. 2).
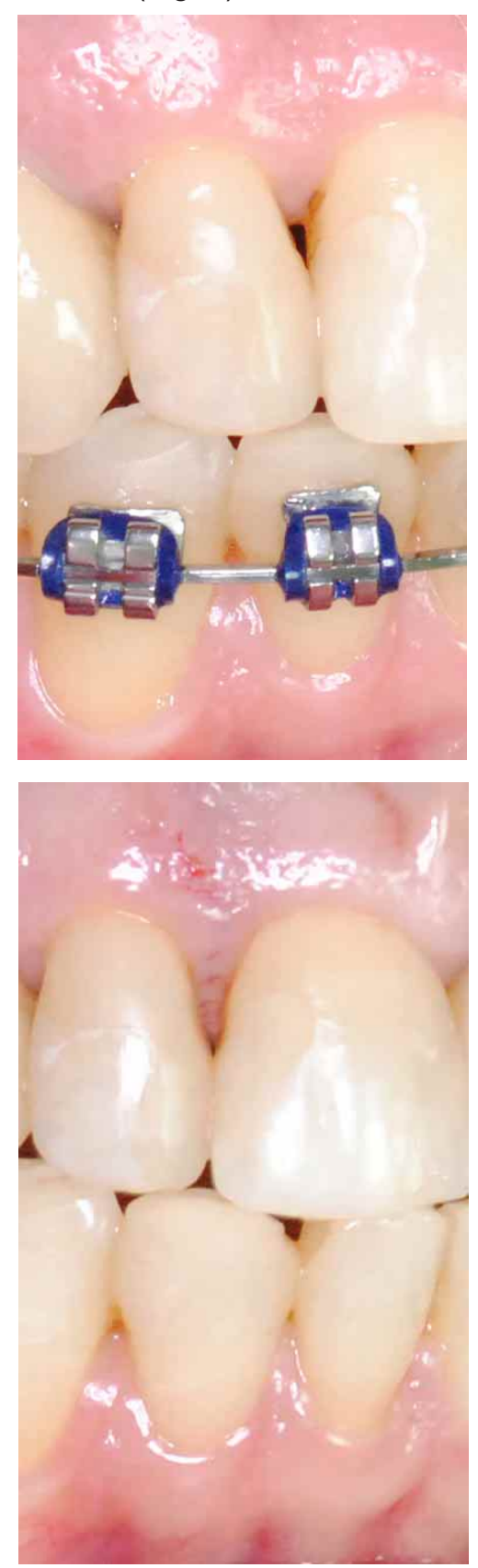

Fig. 2. A) Presencia de espacio negro entre incisivo central y lateral. B) aspecto inmediato, después de la aplicación de AH.
Caso 3: Paciente de sexo masculino 38 años, fue realizada la corrección con ortodoncia para generar espacio en la región del diente 13 y posterior instalación de implantes el que sufrió una recesión y exposición de la zona cervical con visibilidad del metal, generando una queja en el paciente por exposición de la cinta metálica al sonreír. Fue propuesta la aplicación del $\mathrm{AH}$ (TEOSYAL $®$ ), en dos etapas con 21 días de diferencias entre ellas. Los $0,2 \mathrm{~mL}$ se aplicaron en diferentes sectores demostrando buenos resultados posteriores; actualmente se encuentra con 3 años de seguimiento y no fueron necesarias nuevas aplicaciones (Fig. 3).
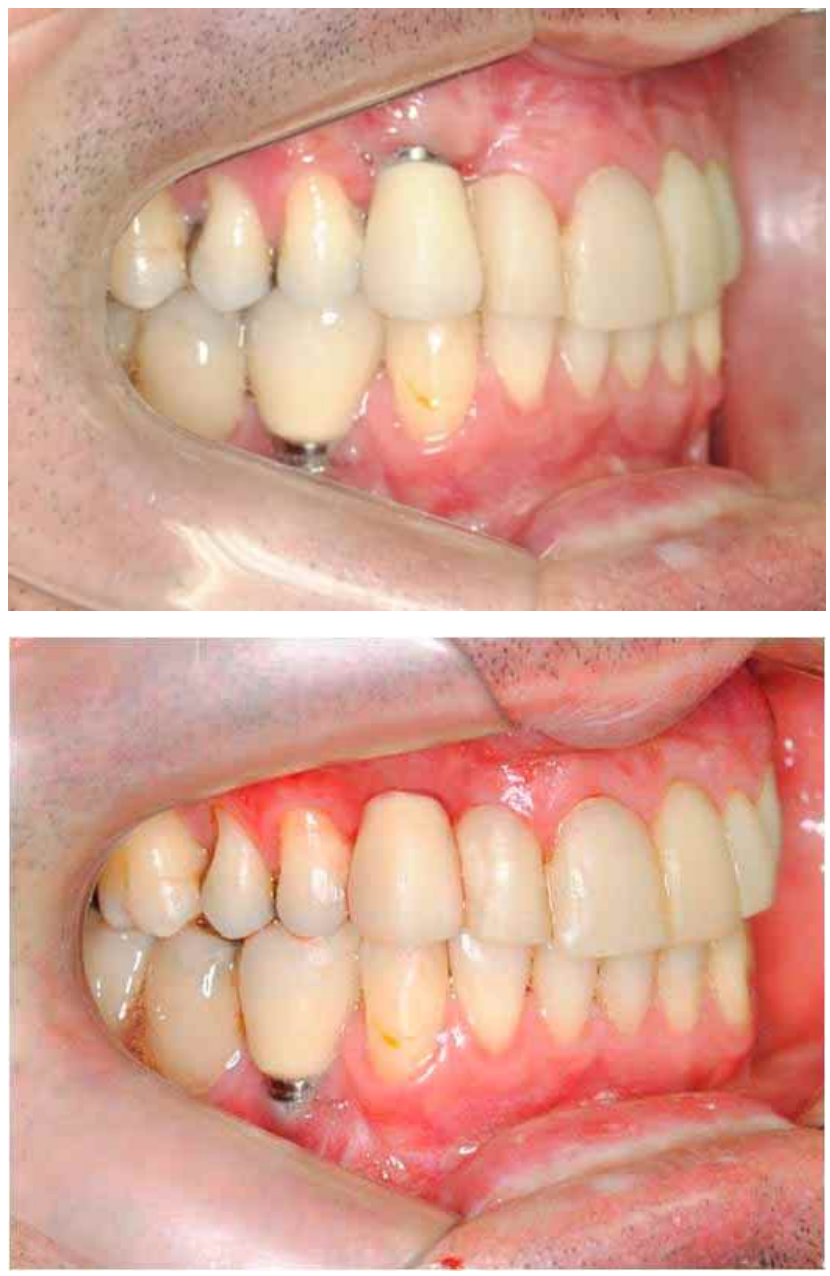

Fig. 3. A) Exposición cervical de implante en zona del diente 13. B) imagen inmediata después de la segunda aplicación de $\mathrm{AH}$.

Caso 4: Paciente de 52 años, sexo masculino, se presentó con exposición cervical de implante instalado en la región del 46; fue propuesto la aplicación de $\mathrm{AH}$ (TEOSYAL ${ }^{\circledR}$ ) en tres aplicaciones intercaladas a cada 21 días, debido a la extensión del defecto, utilizando tres puntos de aplicación de 0,2 mL. Actualmente el 
paciente se encuentra con 3 años de seguimiento y no fueron necesarias nuevas aplicaciones (Fig. 4).
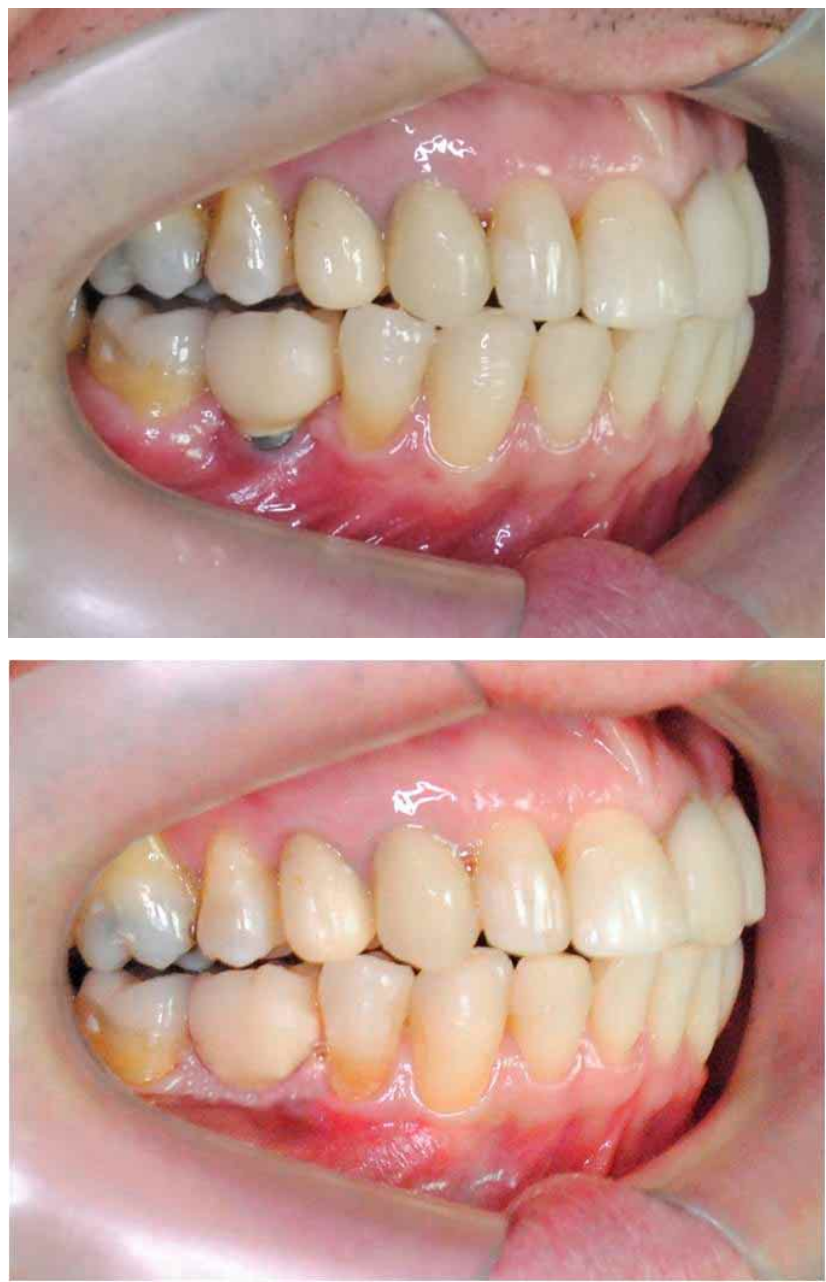

Fig. 4. A) Exposición cervical de implante a nivel del 46. B) imagen con 90 días de seguimiento.

\section{DISCUSIÓN}

La imitación de los dientes naturales, tanto en función como en estética, presentan avances importantes, pero la condición de tejidos blandos en la etapa pre operatoria o postoperatoria puede complicar el escenario final. Debido a la pobre vascularización de las papilas dentales y a la pérdida ósea, muchas técnicas quirúrgicas para la reconstrucción papilar presentan un resultado limitado (Cavalcanti, 2002). El uso del ácido hialurónico puede ser una alternativa para el restablecimiento de la papila interdental, siendo un procedimiento eficaz y previsible sin lesiones o injurias importantes a las estructuras vecinas y sin some- ter al paciente a procedimientos quirúrgicos de larga duración y morbilidad.

Hay muchos factores que determinan el éxito de los procedimientos de recubrimiento radicular como son el biotipo gingival, cantidad de tejido queratinizado, la ubicación del diente en el arco, la prominencia del ancho de la raíz, forma de cepillado dentario y el uso de tabaco (Kumar et al., 2014). En los cuatro casos presentados, los pacientes fueron criteriosamente orientados a mejorar la técnica de cepillado, no eran fumadores y presentaban una calidad gingival adecuada, siendo factores relevantes en el éxito del tratamiento realizado; es claro que la selección del paciente debe ser adecuada y estudios más extensos deben ser realizados para determinar objetivamente estas condiciones.

Engström et al. (2001) investigaron el efecto antiinflamatorio y regenerativo de $\mathrm{AH}$ en defectos periodontales en grupos quirúrgicos y no quirúrgicos. Los autores concluyeron que el $\mathrm{AH}$ en contacto con tejido óseo y blando no tuvo influencia sobre el sistema inmune. Esta conclusión muestra que es un material que puede ser utilizado de forma segura para defectos periodontales menores como en el camuflaje de black spaces y de recesiones gingivales.

Nuestros casos mostraron resultados satisfactorios junto a un evidente aumento del volumen del tejido en todos los casos tratados, restableciendo las condiciones estéticas de los pacientes. Se puede concluir que esta técnica de relleno gingival no quirúrgico puede ser promisoria en la resolución mínimamente invasiva de defectos estéticos periodontales menores, aunque estudios comparativos y secuencias con mayor número de pacientes son necesarios para su indicación adecuada con resultados predecibles.

CELÓRIA, A.; SIGUA-RODRIGUEZ, E. A. \& OLATE, S. Gingival increase based on hyaluronic acid in peri implant and periodontal defects. A case series analysis. Int. J. Odontostomat., 11(4):431-435, 2017.

ABSTRACT: Hyaluronic acid has been used in different anatomical areas, where its application and use for facial rejuvenation as well as joint injuries are known. However, information of its application in periodontal and peri implant defects is limited. The aim of this research is to show the clinical results of a case series in subjects with minor periodontal defects treated with the application of hyaluronic acid. Four subjects with black spaces and gingival recessions were included; the protocol for application of 0.2 
$\%$ hyaluronic acid was realized at different times; the clinical follow-up showed efficiency in the aesthetic recovery and stability in the increases. It is possible to conclude that this minimally invasive technique is promising to treat minor periodontal and peri implant defects.

\section{periodontal defects.}

\section{REFERENCIAS BILIOGRÁFICAS}

Aslan, M.; Simsek, G. \& Dayi, E. The effect of hyaluronic acid-supplemented bone graft in bone healing: experimental study in rabbits. J. Biomater. Appl., 20(3):209-20, 2006. Ballini, A.; Cantore, S.; Capodiferro, S. \& Grassi, F. R. Esterified hyaluronic acid and autologous bone in the surgical correction of the infra-bone defects. Int. J. Med. Sci., 6(2):65-71, 2009.

Cavalcanti, S. M. Reconstrução Cirúrgica de Papilas Interdentais. Monografia de Especialização em Periodontia. Piracicaba, Faculdade de Odontologia de Piracicaba, Universidade Estadual de Campinas, 2002.

Celória, A. Eficiência do Ácido Hialurônico na Regeneração das Papilas Interdentárias e dos Colarinhos Metálicos em Implantes Dentários. In: Cauduri R. Toxina Botulínica \& Preenchedores na Odontologia. Porto Alegre, RGO, 2015. pp.112-21.

Engström, P. E.; Shi, X. Q.; Tronje, G.; Larsson, A.; Welander, U.; Frithiof, L. \& Engstrom, G. N. The effect of hyaluronan on bone and soft tissue and immune response in wound healing. J. Periodontol., 72(9):1192-200, 2001.

Ferguson, E. L.; Roberts, J. L.; Moseley, R.; Griffiths, P. C. \& Thomas, D. W. Evaluation of the physical and biological properties of hyaluronan and hyaluronan fragments. Int. J. Pharm., 420(1):84-92, 2011.

Kassab, M. M. \& Cohen, R. E. The etiology and prevalence of gingival recession. J. Am. Dent. Assoc., 134(2):220-5, 2003.

Kumar, R.; Srinivas, M.; Pai, J.; Suragimath, G.; Prasad, K. \& Polepalle, T. Efficacy of hyaluronic acid (hyaluronan) in root coverage procedures as an adjunct to coronally advanced flap in Millers Class I recession: A clinical study. J. Indian Soc. Periodontol., 18(6):746-50, 2014.

\author{
Dirección para correspondencia \\ Eder Alberto Sigua Rodriguez \\ Dirección: Centro de Investigaciones del Colegio \\ Odontológico ( $\mathrm{ClCO})$ \\ Institución Universitaria Colegios de Colombia \\ Bogotá \\ COLOMBIA
}

E-mail: edersiguaodont@gmail.com

Recibido : 28-09-2017

Aceptado: 11-11-2017 